Original Research Paper

\title{
Laser-Induced Breakdown and Detonation in Particle-Laden Systems
}

\author{
${ }^{1,2}$ Konstantin Volkov \\ ${ }^{I}$ Saint Petersburg National Research University of Information Technologies, Mechanics and Optics, St Petersburg, Russia \\ ${ }^{2}$ Faculty of Science, Engineering and Computing, Kingston University, London, UK
}

Article history

Received: 18-08-2014

Revised: 19-11-2014

Accepted: 26-01-2015

\begin{abstract}
Simulation of the interaction of a laser pulse with gas-droplet and gas-particle mixtures plays an important role in environmental and engineering applications. The injection of liquid droplets or metal particle causes optical breakdown on the individual droplet or particle and leads to a drop of the detonation minimum pulse energy of the mixture. Mathematical models of various stages of the optical breakdown are developed and applied to the analysis of laser-induced breakdown in particle-laden systems. The numerical calculations are based on an in-house computer code using the finite-volume method and high-resolution scheme. The threshold of optical breakdown and its dependence on contributing factors (droplet radius, location of droplet, total energy and time of laser pulse, radius of laser spot) has been studied. Minimum pulse energy of detonation was computed in the hydrogen-oxygen mixture with metal particles and liquid droplets.
\end{abstract}

Keywords: Droplet, Laser Pulse, Optical Breakdown, Detonation, Numerical Simulation

\section{Introduction}

Interaction of a laser pulse with gas-particle and gasdroplet mixtures plays an important role in different applications. These include environmental monitoring of high-risk industrial objects and enclosed spaces (coal mines, oil storages and others), measurements of flammability and explosion limits in particulate reacting substances and propagation of laser radiation through explosive mixtures as well as laser-induced volumetric explosion for application to fire mitigation and the design of air-breathing pulse detonation engines. Use of a laser pulse allows the creation of desired temporal and spatial distributions of ignition centers to perform a homogeneous ignition within sub-microsecond intervals. Laser ignition has the potential to replace the conventional electric spark plugs in engines that are required to operate under much higher compression ratios, faster compression rates and much leaner fuel-to-air ratios.

Vaporization of droplets by absorption of laser radiation is also of practical interest for many reasons, such as communication through the medium, transmission of optical images and clearing the dropletdebris from an area. Laser propulsion using water droplets as a propellant is considered to have a wide range of future applications in space. In order to have a more efficient propulsive effect with a minimum consumption of water, further studies on laser-droplet interactions have become more important. There are similarities between the laser-induced acoustic shock waveforms and those associated with blast waves and sonic booms. The acoustic pulses associated with laserinduced sparks could be used in the laboratory to simulate blast sounds from explosions or sonic booms and to investigate the associated propagation effects.

When a high-power laser pulse $\left(I_{*} \sim 10^{11} \mathrm{~W} / \mathrm{cm}^{2}\right)$ is focused at a point and interacts with a gas, the gas is heated to temperatures of thousands of degrees within several microseconds, breaking down and becoming highly ionized (Kopecek et al., 2003; Ma et al., 1998). This process is always accompanied by a light flash and generation of sound (acoustic shock wave).During optical breakdown, a very high free electron density (plasma) of the order of $10^{18}-10^{20}$ electron $/ \mathrm{cm}^{3}$ is produced. Two mechanisms are responsible for laserinduced optical breakdown: Multi-photon ionization and cascade ionization (avalanche or impact ionization). For long pulses, breakdown is primarily caused by avalanche ionization, while multi-photon absorption dominates breakdown in the low regime (Fan et al., 2002). Both multi-photon absorption and avalanche ionization depend on the laser intensity in the interaction volume and both require minimum threshold intensity before breakdown is initiated (Musing et al., 2007). 
Particles or droplets, trapped by a laser beam, considerably influence results of the optical breakdown (Armstrong and Zardecki, 1990). The injection of metal particles with low evaporation temperature and low ionization potential (e.g., aluminum) leads to a drop of detonation minimum pulse energy $\left(I \sim 10^{9} \mathrm{~W} / \mathrm{cm}^{2}\right)$ due to optical breakdown on individual particles. Vapor aureole around metal particle is a source of free electrons and optical breakdown in the gas-particle mixture requires a lower energy laser pulse than in a pure gas (Chang et al., 1988).

Much work has been performed on the interaction between single particles and droplets and focused laser beams demonstrating the influence of the liquid and droplet size on the breakdown process (Armstrong and Zardecki, 1990; Emelyanov et al., 2003; Emelyanov and Volkov, 2006; Papalexandris, 2004; Volkov and Emelyanov, 2012). It has been shown that the droplet acts like a lens and focuses the laser light with ignition occurring on the shadow side of the droplet in either the gas or liquid phase. Once breakdown has occurred, plasma is vented from the droplet away from the laser and explosive vaporization of the droplet occurs. The breakdown energy is a function of droplet and gas material and of droplet size and this is over two orders of magnitude smaller than for gas phase ignition.

The explosive vaporization of a single aerosol droplet by pulsed laser radiation is investigated by (Carls and Brock, 1987). The slow vaporization regime considered incorporated molecular transport effects but assumes a constant pressure field, thus eliminating convection. Breakdown was not considered. However, the results obtained supported the possibility of ionization in the shock-heated air.

A model for simulating the laser-induced breakdown in air and behind droplets is developed by (Musing et al., 2007). The model was based on laser energy absorption by inverse bremsstrahlung and multi-photon ionization. Simulations, as well as experimental results, were presented, varying the gas pressure, laser pulse length, focal spot size of the focusing lens and droplet size of the parameters. A physical model describing the process of the laser and water droplet interaction was developed by (Emelyanov and Volkov, 2006).

Processes that control transport and combustion of particles and droplets remain unresolved and introduce significant uncertainties into modeling and simulation. One of the most important parameters for practical applications is the minimum pulse energy required to induce ignition and detonation of the mixture. Physical and mathematical models of optical breakdown on individual particle and droplet and numerical methodology for computer modeling are developed in this study. Minimum pulse energy of detonation is computed for mixtures of various compositions.

\section{Laser Pulse}

The intensity of the laser pulse is represented as a product of the centerline intensity, $I_{m 0}$, the function describing the time distribution of the intensity, $f_{1}(t)$, the function taking into account the spatial distribution of the intensity, $f_{2}(r)$ and the function describing absorption of radiation in the medium, $f_{3}(z)$. The intensity of the laser pulse is:

$$
\mathrm{I}(\mathrm{t}, \mathrm{r}, \mathrm{x})=\mathrm{I}_{\mathrm{m} 0} \mathrm{f}_{1}(\mathrm{t}) \mathrm{f}_{2}(\mathrm{r}) \mathrm{f}_{3}(\mathrm{z})
$$

where, $t$ is time, $r$ is radial coordinate and $z$ is the coordinate indicating the direction of propagation of the laser beam.

The theoretical peak intensity of the laser pulse at any radial point is calculated for a given power and degree of focus. The laser does not reach its peak operating power at the moment when it is turned on. It requires a short time to ramp up to its peak output. For a laser pulse which lasts $8 \mu \mathrm{s}$, the laser output reaches its peak intensity in about one fourth of a pulse duration and will have dropped to roughly three fourth of its peak value when the laser is shut off. The laser model includes a ramp time parameter during which time the laser's output increases linearly to a maximum (Fig. 1).

The time distribution of the intensity is represented by a continuous piecewise-linear function:

$$
f_{1}(t)=\sum_{k=1}^{N-1}\left[F_{k}+\left(F_{k+1}-F_{k}\right) \frac{t-t_{k}}{t_{k+1}-t_{k}}\right] \phi\left(t_{k}, t_{k+1}\right)
$$

where, $t_{k}$ and $I_{k}$ are time and intensity of the laser pulse and $N$ is the number of ramp points. The function $\varphi\left(t_{k}\right.$, $\left.t_{k+1}\right)$ is given by:

$$
\phi\left(t_{k}, t_{k+1}\right)=\frac{t-t_{k}+\left|t-t_{k}\right|}{2\left|t-t_{k}\right|+\varepsilon}-\frac{t-t_{k+1}+\left|t-t_{k+1}\right|}{2\left|t-t_{k+1}\right|+\varepsilon}
$$

where, $\varepsilon$ is the small value used to avoid division by zero.

A continuous piecewise-linear representation of the pulse shape is used to compute the temporal characteristic:

$$
\mathrm{S}_{\Sigma}=\int_{0}^{\infty} \mathrm{f}_{1}(\mathrm{t}) \mathrm{dt}=\frac{1}{2} \sum_{\mathrm{k}=1}^{\mathrm{N}-1} \frac{\mathrm{F}_{\mathrm{k}+1}+\mathrm{F}_{\mathrm{k}}}{\mathrm{t}_{\mathrm{k}+1}-\mathrm{t}_{\mathrm{k}}}
$$

In a plane normal to the direction of laser pulse, the spatial distribution of the intensity is described by the normal distribution (Fig. 2):

$f_{2}(r)=\exp \left(-\frac{2 r^{2}}{R^{2}}\right)$

where, $r$ is the radial distance from the centerline of the beam and $R$ is the radius of the laser spot. 


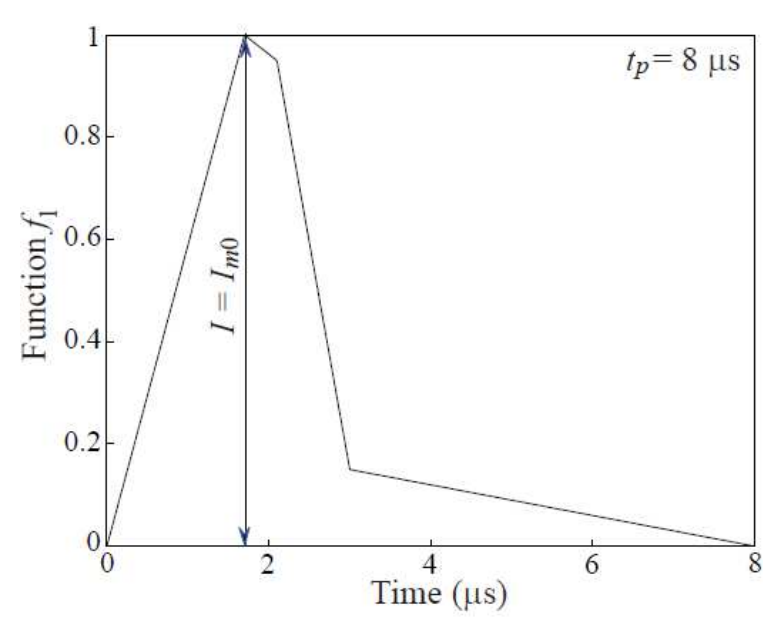

Fig. 1. Intensity of laser pulse as a function of time

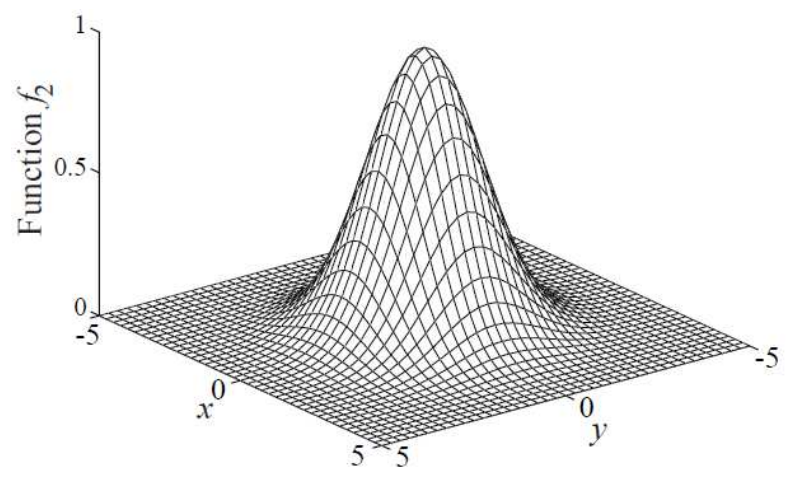

Fig. 2. Intensity of laser pulse as a function of radial coordinate (radius of laser spot is $5 \mathrm{~mm}$ )

The absorption of radiation is described by BouguerLambert-Beer law:

$$
\mathrm{f}_{3}(\mathrm{z})=\exp (-\mathrm{kz})
$$

where, $k$ is the absorption coefficient depending on the nature, state and fraction of particles as well as on the wave length of laser radiation.

The total energy of the laser pulse is related to its intensity:

$$
\mathrm{Q}_{\Sigma}=\int_{0}^{\infty} \int_{0}^{2 \pi} \int_{0}^{\infty} \mathrm{I}_{\mathrm{m} 0} \mathrm{f}_{1}(\mathrm{t}) \mathrm{f}_{2}(\mathrm{r}) \mathrm{rdrd} \phi \mathrm{dt}
$$

where, $\varphi$ is a polar angle.

\section{Breakdown Mechanisms}

The physical model of the optical breakdown provides a qualitative description of the processes, in particular the interaction of the laser pulse with individual metal particles and liquid droplets.

\section{Metal Particle}

A chain of processes leading to explosion and optical breakdown of an individual metal particle was developed (Fig. 3). These processes depend on the optical properties of the particle, its shape and ratio of particle size to radius of the laser spot.

The particle is heated up to a high temperature, melting and evaporation start (Fig. 3a). Evaporation of the particle leads to the formation of a vapor aureole around it. Free electrons are generated in the vapor aureole as a result of thermal emission from the particle surface (if $T<T_{b}$ ) and isothermal ionization in the vapor aureole (if $T>T_{b}$ ). This leads to collisions of electrons with ions and atoms and electron-electron collisions. Ionization of the vapor aureole due to reverse drag effect leads to the development of an electron avalanche and formation of micro-plasma spots around the particle (Fig. 3b). The cascade ionization process is significant at high pressure and longer laser pulse because, under these conditions, electron-atom or electron-ion collisions have sufficient time to occur during the laser pulse (Kopecek et al., 2003). Micro-plasma spots are expanded due to thermal diffusion of electrons and ionization of molecules and atoms of the surrounding as. Micro-plasma spots are merged and a plasma fireplace is formed around the ensemble of particles (Fig. 3c). The plasma fireplace absorbs laser radiation and contributes to the development and propagation of a self-sustaining shock wave in the gas-particle mixture (Fig. 3d).

\section{Liquid Droplet}

The high-intensity laser beam interacts with a single droplet with radius much larger than the input laser wavelength.

Compared to a metal particle, heating and evaporation of a liquid droplet are delayed due to weak absorption of the laser radiation. Concentration of free electrons in the vapor aureole is insufficient for development of an electron avalanche. In this case, the key mechanism of development of optical breakdown is explosive evaporation of the droplet (Fig. 4).

The laser radiation focuses inside a droplet near its shadow side (Fig. 4a). In this region, overheating conditions arise and the liquid is in a meta-stable state in which its temperature exceeds the temperature of the saturated vapor at a given temperature. An internal vapor cavity is formed and the liquid boils off in this cavity (Fig. 4b). Increase in pressure in the vapor cavity creates conditions for internal micro-breakdown. An internal micro-plasma spot appears and absorbs the laser radiation (Fig. 4c). Further increase in pressure in the vapor cavity forms a shockwave expanding inside the droplet (Fig. 4d). Expansion of this shock wave induces thermal ionization of the surrounding gas on the shock wave front (Fig. 4e). Free electrons that have appeared on the shock wave front induce chain mechanism of breakdown, receiving their energy due to the reverse drag effect (Fig. 4f). 


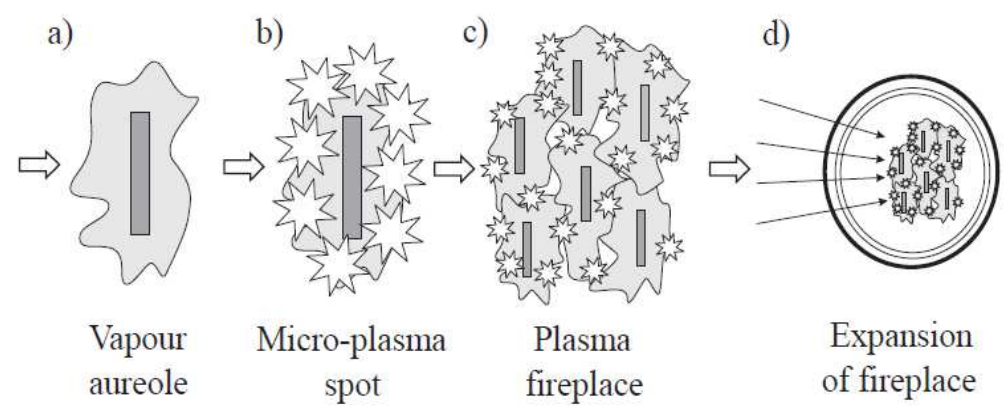

Fig. 3.Optical breakdown on a metal particle
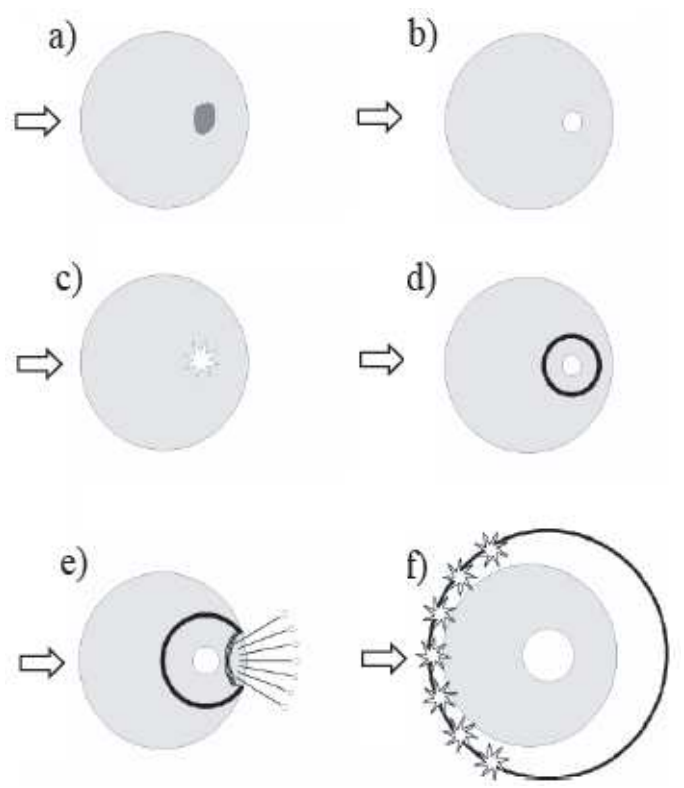

Fig. 4. Optical breakdown on a liquid droplet

Intense vaporization of the droplet leads to the thermal destruction of the droplet either by means of local jetting of a part of the droplet mass or by its explosion.

\section{Mathematical Model}

The problem considered is multi-physical and multi-scale. To predict the properties of multi-phase mixture, a mathematical model is formulated based on a two-level approach.

\section{Two-Level Approach}

The mathematical formulation of the problem is divided into low-level and high-level models. Low-level models correspond to the processes in the volume occupied by an individual particle or droplet. The highlevel models correspond to the processes in the volume occupied by the multi-phase mixture. The chain of events leading to the optical breakdown on the individual particle and volume detonation of the gas-particle mixture is shown in the Fig. 5.
The model of multi-velocity and multi-temperature continuum is used to formulate equations describing the detonation of a gas-particle mixture. The numerical solution is based on the finite volume method and the splitting scheme for the physical processes.

\section{Low-Level Models}

Low-level models describe heating, evaporation and formation of the vapor aureole, appearance of free electrons due to thermal ionization on the front of the shock wave and development of the electron avalanche due to the reverse drag effect.

To compute the optical properties of the particle (e.g., absorption efficiency of laser radiation), semiempirical data are used. There are detailed data about temperature dependencies of optical, thermal and physical properties of aluminum because it often occurs in practice. The heating model is based on a numerical solution of the unsteady heat diffusive equation (Astafieva and Prishivalko, 1998). The equations describing the electron avalanche in the vapor aureole include those of heating of the vaporaureole due to electron-atom collisions, the warming-up of the electrons, the ionization kinetic equation of vapor as a result of electron impact and the equation of particle mass. The plasma in the vapor aureole is considered as an ideal gas. The Euler equations are used for the simulation of the gas dynamical processes in the vapor aureole. A simple model of a one-step chemical reaction is used in order to reproduce the explosion of an individual particle.

A high-power laser pulse that is focused into a droplet produces a vapor cavity. A mathematical model for the spherically symmetric motion of a laserinduced bubble is used which accounts for gas and liquid compressibility, heat and mass transport effects inside bubble and liquid (Emelyanov et al., 2003).

The threshold value of optical breakdown on an individual particle and droplet is computed as a result of the solution of low-level models. Low-level models are incorporated in high-level models that describe detonation of the mixture. 


\section{High-Level Models}

The multi-phase mixture consists of some gas and particulate components (Fig. 6). The gas phase consists of a combustible component (fuel), an oxidant component, a component that is the combustion products and a neutral component. Particulate phase represents an ensemble of metal particles and liquid droplets. Vapor phase is a product of thermal decomposition of metal particles consisting of atoms, ions and electrons.
Condensed phase represents the metal oxide that is a result of vapor condensation.

The Eulerian approach is used to formulate the governing equations describing the detonation of the mixture. These are written for all gas and particulate components of the mixture. The diffusion processes are ignored because they define the evolution of the mixture on the time scales, which are much longer than the time of the laser pulse.

Independent models

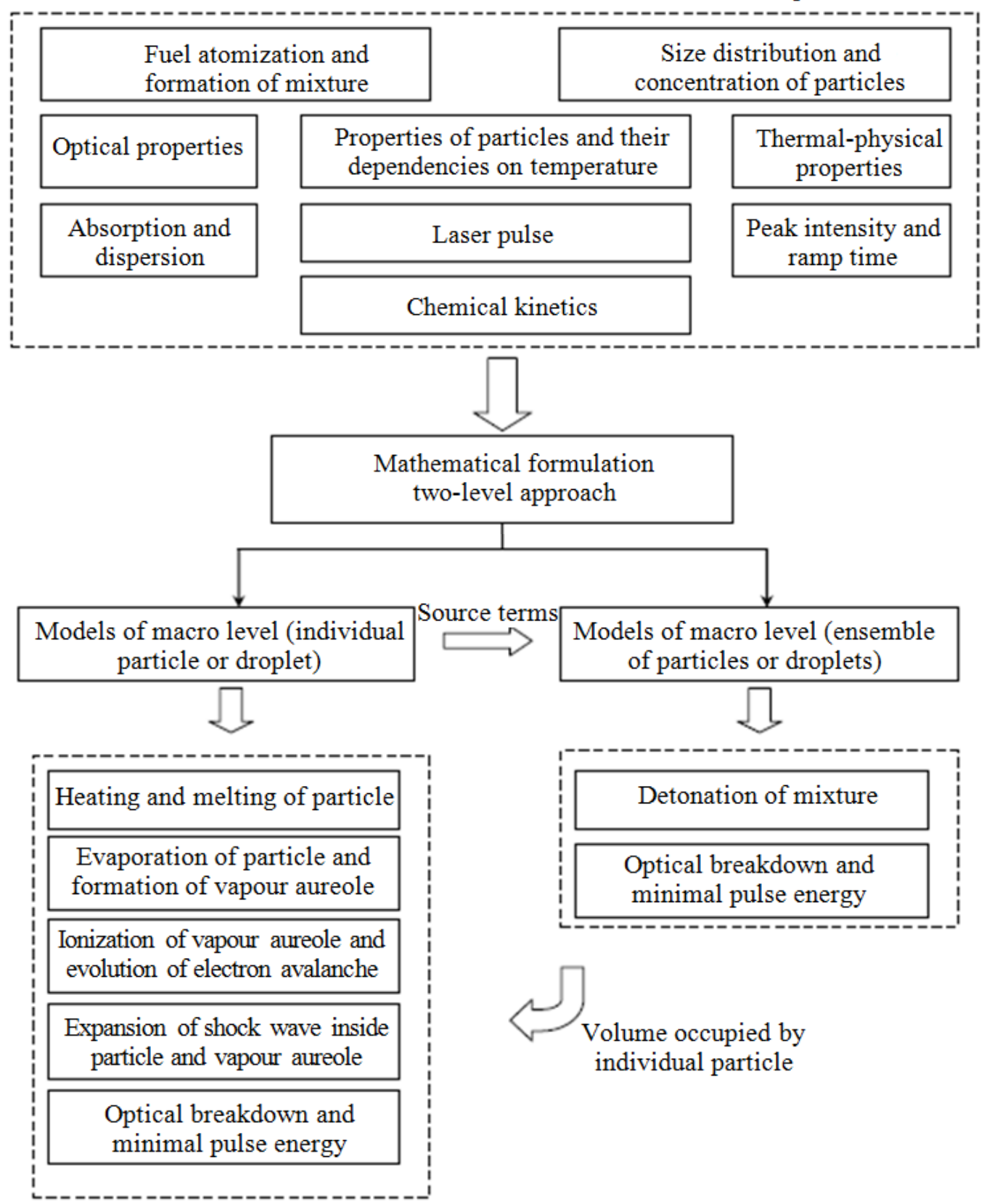

Fig. 5. Two-level approach for simulation of laser-induced detonation in a multi-phase mixture 


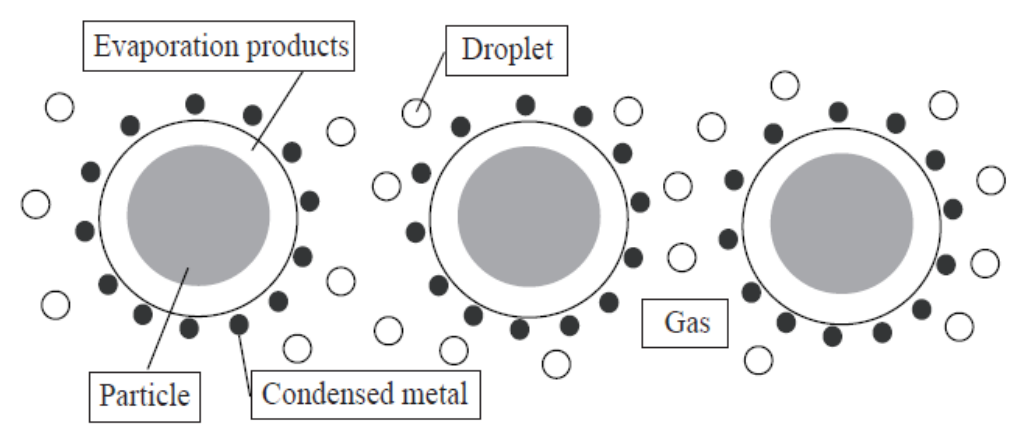

Fig. 6. Composition of the multi-phase mixture

The data obtained from the solution of the lowlevel problems are used to calculate source terms in the governing equations describing the high-level problem. It is assumed that particles are uniformly distributed in the domain. Some volume of the mixture depending on the particle volume fraction is associated with each particle (individual reactor of a particle). The model of an unsteady, well-stirred reactor is used to calculate the physical quantities in this volume. The solution of the high-level problem provides the fraction of particles and the volume occupied by a particle.

\section{Computational Procedure}

An in-house CFD computer code has been developed. The equations are solved numerically based on the finite volume method, splitting scheme on the physical factors, a piecewise parabolic method and Chakravarthy-Osher scheme for in viscid fluxes. Approximate Riemann solver is employed to calculate the interfacial fluxes on the first fractional time step. Chemical reactions and interphase exchange of mass, momentum and energy are considered at the second fractional time step. The internal time step is used to ensure stability of the numerical scheme. A pseudogas of particles does not have an internal pressure, so an artificial pressure is introduced to design similar computational procedures for gas and particulate components.

\section{Results}

The physical model of the optical breakdown provides a qualitative description of the processes, in particular the interaction of the laser pulse with individual metal particles and liquid droplets.

\section{Metal Particle}

Figure 7 shows heating of a metal particle up to the boiling temperature. The particle temperature depends on the total energy of the laser pulse and the distance from particle to centerline of the laser beam.
The degree of ionization as function of time and total energy of the laser pulse is shown in Fig. 9. Micro-plasma spots around the particle are formed at an energy of $1.03 \mathrm{~J}$. Pre-breakdown conditions are sensitive to a small change of energy of the laser pulse. The threshold value of plasma formation is defined as a proportion of the power used up to the beginning of breakdown.

Development of an electron avalanche in the form of its dependence on the degree of ionization of the vapor aureole with time is shown in the Fig. 8. The particle is located on the centerline of the laser beam.

\section{Liquid Droplet}

The temperature field in a water droplet and surrounding is shown in Fig. 10. The laserbeam falls on the droplet from right to left. A local temperature rise is observed on the exposed surface of the droplet. A thin thermal boundary layer is formed in the vicinity of the droplet. An increase in temperature inside the droplet corresponds to the center of an internal vapor cavity which is located near the shadow side of the droplet. The droplet is superheated and water is in the meta-stable state. Line 7 corresponds to the start of the explosive process at a temperature of $698 \mathrm{~K}$. The time of explosive transformation of a droplet is $1.54 \mu \mathrm{s}$.

The plasma spot is non-transparent to radiation. An increase in pressure and temperature induces the expansion of a shock wave. Its intensity decreases with increase in distance from the center of the vapor cavity. Table 1 and 2 show optical breakdown conditions depending on the droplet radius and energy of the laser pulse. The first column corresponds to the energy of laser pulse. The second column indicates the time of explosive transformation. The third column gives the value of the degree of ionization. The fourth column indicates validation of the condition of explosive transformation. The fifth column indicates the presence or absence of an electron avalanche. 


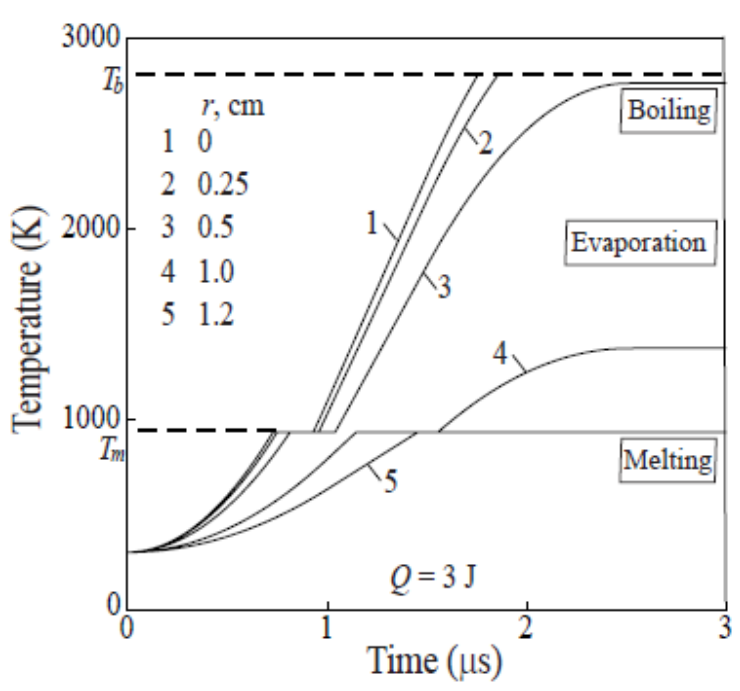

Fig. 7. Temperature of the metal particle

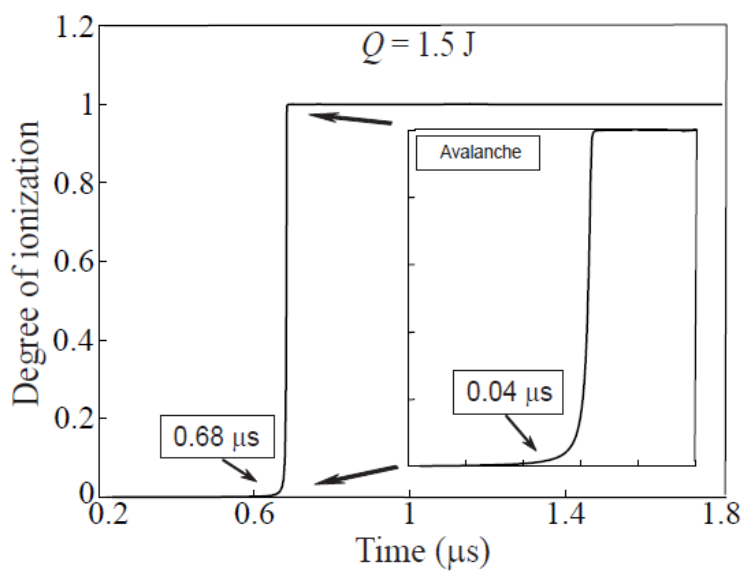

Fig. 8. Development of electron avalanche

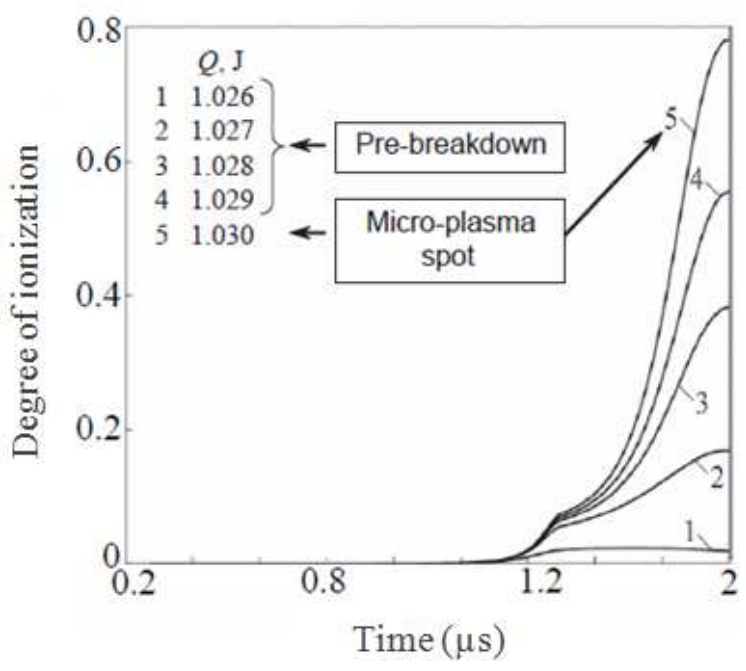

Fig. 9. Degree of ionization of vapor aureole

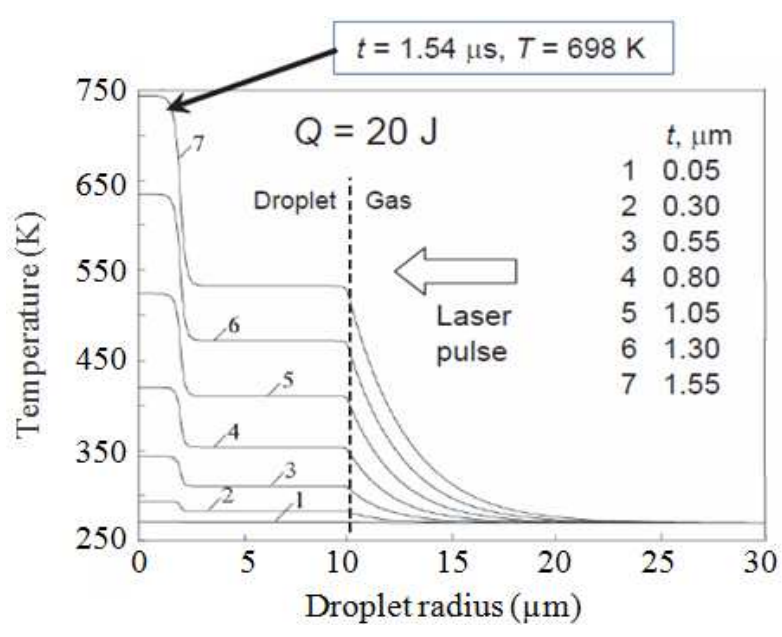

Fig. 10. Temperature field inside and outside droplet

Table 1. Optical breakdown conditions for $\mathrm{rp}=5 \mu \mathrm{m}$

\begin{tabular}{lllll}
\hline$Q_{\Sigma}, \mathrm{J}$ & $t_{e}, \mu \mathrm{s}$ & $\alpha$ & $T>T_{e}$ & Yes/No \\
\hline 5 & - & - & - & - \\
10 & 1.83 & $10^{-2}$ & + & + \\
15 & 1.38 & $10^{-2}$ & + & + \\
20 & 1.13 & $10^{-2}$ & + & + \\
30 & 0.93 & $10^{-2}$ & + & + \\
50 & 0.72 & $10^{-2}$ & + & + \\
\hline
\end{tabular}

Table 2. Optical breakdown conditions for $\mathrm{rp}=15 \mu \mathrm{m}$

\begin{tabular}{lllll}
\hline$Q_{\Sigma}, \mathrm{J}$ & $t_{e}, \mu \mathrm{S}$ & $\alpha$ & $T>T_{e}$ & Yes/No \\
\hline 5 & - & - & - & - \\
10 & - & - & - & - \\
15 & 1.48 & $10^{-8}$ & + & - \\
20 & 1.23 & $10^{-8}$ & + & - \\
30 & 1.04 & $10^{-8}$ & + & + \\
50 & 0.83 & $10^{-8}$ & + & + \\
\hline
\end{tabular}

\section{Minimum Pulse Energy}

One of the most important parameters for laser ignition applications is the minimum laser pulse energy for ignition (MPE) and its dependence on the pressure of the combustible gas mixtures. It is of critical importance in determining the laser conditions at which a spark is produced. Knowledge of these conditions is important not only a for fundamental understanding of the ignition process but also for the selection of lasers, optics windows and beam delivery systems for the design of a practical laser ignition system.

The minimum pulse energy is a function of the radius of the laser spot, mass fraction of particulate components and volume fraction of the oxidant.

The results obtained are presented in the Fig. 11 for fish-plate aluminum particles in the acetylene-oxygennitrogen mixture. The volume fraction of acetylene is $15 \%$, that of oxygen changes from 15 to $35 \%$. The mass fraction of particles is $1 \mathrm{~g} / \mathrm{m}^{3}$. 


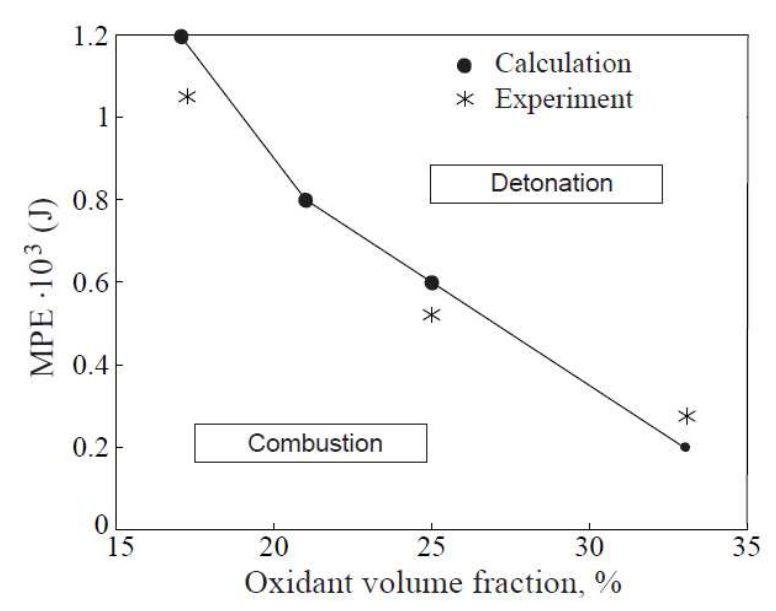

Fig. 11. Minimum pulse energy of the detonation

The wave length of the laser beam is $4.2 \mu \mathrm{m}$, radius of the laser spot is $1.5 \mathrm{~cm}$ and the time of pulse is $2.6 \mu \mathrm{s}$ (Aleksandrov et al., 2005; Azarov et al., 2004).

At $Q=150 \mathrm{~J}$, combustion of fuel takes place in a small region adjacent to the shock wave front. At $Q=$ $200 \mathrm{~J}$, the temperature and pressure in the shock wave front increase and the volume fraction of fuel decreases by $20-30 \%$ for the time of the laser pulse. Further energy supply to the mixture leads to a considerable increase in the temperature and pressure in the mixture and development of unsteady gas dynamic processes in the vapor aureole. At $Q=300 \mathrm{~J}$, about $60 \%$ of the fuel is used and at $Q=300 \mathrm{~J}$, about $95 \%$ of the fuel burns beyond the shock wave front. The energy of the laser pulse, 350-400 J, is the MPE of the detonation.

\section{Discussion}

Irradiance with light flux intensities of about $10^{11}$ $\mathrm{W} / \mathrm{cm}^{2}$ leads to gas breakdown and high ionization of the medium. In laser-induced plasma, inverse bremsstrahlung absorption leads to an increase in electron energy. Electrons are able to ionize the carrier gas and form an exponentially growing electron cascade. The electron avalanche is developed in $0.68 \mu$ s from the laser pulse started and ionization takes place within a short time interval (about $0.04 \mu \mathrm{s}$ ).

Interaction of the laser pulse with an individual metal particle is related to one of the following stages:

- $\quad$ Pre-threshold energy of the laser pulse $(Q<1 \mathrm{~J})$. Energy of the laser pulse is not enough to ionize the vapor aureole around the particle. Evaporation of the particle exists but the degree of ionization is small and the vapor aureole is transparent for laser radiation

- Near-threshold energy of the laser pulse $(Q=1-2$ $J)$. Degree of ionization changes from some percent to $100 \%$
- $\quad$ Post-threshold energy of the laser pulse $(Q>2 \mathrm{~J})$

The process proceeds for a completely ionized vapor aureole around the particle. Ionization has an avalanche character within a short time interval.

The time of optical breakdown is a result of the competition between 3 factors: (i) Time of droplet heating to the temperature of explosive transformation (at a low pulse energy, the large droplets do not have enough time for being heated and the small droplets exchange heat intensively with the surrounding), (ii) intensity of the shock wave contributing to the thermal ionization of the vapor (for massive droplets, the shock wave is weak), (iii) time of development of an electron avalanche.

The size of the laser spot has a significant impact on the threshold value of the optical breakdown. For droplets of $5 \mu \mathrm{m}$, breakdown takes place at energy of 10 $\mathrm{J}$. Increase in droplet radius leads to an increase in the time of droplet heating and decrease in the degree of ionization. No electron avalanche develops at low intensity of the laser pulse and the threshold value of optical breakdown increases.

In the explosive vaporization regime, molecular transport effects such as mass diffusion and heat conduction are small compared to convective transport. Convective transport is driven by gradients in the pressure field. Convection becomes important when the vapor pressure of the evaporating droplet becomes comparable to the pressure of the surrounding gas. Under these conditions, the rate of evaporation becomes greater than the rate at which diffusion can conduct mass away from the droplet. As the temperature and, therefore, the vapor pressure and evaporation rate of the droplet rise, convection becomes an effective mechanism for mass transport away from the droplet. At higher temperatures, it becomes the dominant mechanism.

\section{Conclusion}

Laser-induced breakdown in the carrier gas and behind droplets has been investigated numerically. The chain of events leading to the optical breakdown on individual metal particles of non-spherical shape, liquid droplets and detonation of a gas-particle mixture has been developed. The spark formation is described by the inverse bremsstrahlung absorption and subsequent collisional ionization. Results of the simulation quantify the minimum laser intensity necessary for breakdown.

The mathematical formulation of the problem was divided into low-level models and high-level models. The low-level models correspond to the processes in the volume occupied by an individual particle or droplet. The high-level models correspond to the processes in the volume occupied by the multi-phase mixture. The data 
obtained from the solution of the low-level problems are used to calculate source terms in the governing equations describing the high-level models. The solution of the high-level model provides the volume fraction of particles and the volume occupied by an individual particle or droplet.

Models of consistent stages of optical breakdown on metal particles and liquid droplets have been developed. These stages include heating and evaporation, formation of a vapor aureole, generation of free electrons due to thermal ionization beyond the shock wave front, development of electron avalanche due to the reverse drag effect and gas dynamic processes in the vapor aureole and the surroundings.

The threshold intensities of optical breakdown and minimum pulse energy of detonation in multi-phase mixture have been computed and contributing factors have been studied.

\section{Acknowledgement}

The author wishes to thank colleagues from the Russian Scientific Centre for Applied Chemistry (St Petersburg, Russia) for experimental data and discussion of the computational results.

\section{Author's Contributions}

The author developed mathematical models and performed numerical calculations.

\section{Ethics}

This article is original and contains unpublished material. The corresponding author confirms that all of the other authors have read and approved the manuscript and no ethical issues involved.

\section{References}

Aleksandrov, B.S., E.A. Klimuk, K.A. Kutumov, B.M. Lacour and V. Puech et al., 2005. A repetitively pulsed HF laser with a large discharge gap operating on the $\mathrm{F}_{2}-\mathrm{H}_{2}$ mixture. Quantum Electronics, 35: 805-808. DOI: $10.1070 /$ Q2005v035n09ABEH0 10350

Armstrong, R.L. and A. Zardecki, 1990. Propagation of high energy laser beams through metallic aerosols. Applied Optics, 29: 1786-1792.

DOI: $10.1364 / A O .29 .001786$

Astafieva, L.G. and A.P. Prishivalko, 1998. Heating of solid aerosol particles exposed to intense optical radiation. Int. J. Heat Mass Transfer, 41: 489-499. DOI: 10.1016/S0017-9310(97)00108-7
Azarov, M.A., E.A. Klimuk, K.A. Kutumov, G.A. Troshchinenko and B.M. Lacour, 2004. Pulsed chemical HF laser with a large discharge gap. Quantum Electronics, 34: 1023-1026. DOI: 10.1070/QE2004v034n11ABEH002692

Carls, J.C. and J.R. Brock, 1987. Explosion of a water droplet by pulsed laser heating. Aerosol Sci. Technol., 7: 79-90.

DOI: $10.1080 / 02786828708959148$

Chang, R.K, J.H. Eickmans, W.F. Hsieh, C.F. Wood and J.Z. Zhang et al., 1988. Laser-induced breakdown in large transparent water droplets. Applied Optics, 27: 2377-2385. DOI: $10.1364 /$ AO .27 .002377

Emelyanov, V., L. Solong and K. Volkov, 2003. Heat and mass transfer in gas-disperse systems exposed to intense radiation. Heat Transfer Res., 34: 38-50. DOI: 10.1615/HeatTransRes.v34.i5-6.50

Emelyanov, V.N. and K.N. Volkov. 2006. Modelling of the interaction of a pulse of laser radiation with a liquid droplet. J. High Temperature Material Process., 10: 141-159.

DOI: 10.1615/HighTempMatProc.v10.i1.100

Fan, C.H., J. Sun and J.P. Longtin, 2002. Breakdown threshold and localized electron density in water induced by ultrashort laser pulses. J. Applied Phys., 91: 2530-2536. DOI: 10.1063/1.1433929

Kopecek, H., H. Maier, G. Reider, F. Winter and E. Winther, 2003. Laser ignition of methane-air mixtures at high pressures. Experimental Thermal Fluid Sci., 27: 499-503. DOI: $10.1016 / \mathrm{S} 0894-1777(02) 00253-4$

Ma, J.X., D.R. Alexander and D.E. Poulain, 1998. Laser spark ignition and combustion characteristics of methane-air mixtures. Combustion Flame, 112: 492-506. DOI: $10.1016 / \mathrm{S} 0010-2180(97) 00138-7$

Musing, A., U. Riedel, J. Warnatz, W. Herden and H. Ridderbusch, 2007. Laser-induced breakdown in air and behind droplets: A detailed Monte-Carlo simulation. Proc. Combustion Institute, 31: 3007-3014. DOI: 10.1016/j.proci.2006.07.185

Papalexandris, M.V., 2004. Numerical simulation of detonations in mixtures of gases and solid particles. J. Fluid Mechanics, 507: 95-142. DOI: $10.1017 / \mathrm{S} 0022112004008894$

Volkov, K. and V. Emelyanov, 2012. Interaction of Laser Pulse with Liquid Droplet. In: 28th International Symposium on Shock Waves, Kontis, K., (Ed.), Springer Science and Business Media, Berlin, ISBN-10: 3642256880, pp: 265-272. 